\title{
Extended range of a gun launched smart projectile using controllable canards
}

\author{
Mark Costello \\ Department of Mechanical Engineering, Oregon State \\ University, Corvallis, OR 97331, USA
}

\begin{abstract}
This effort investigates the extent to which moveable canards can extend the range of indirect fire munitions using both projectile body and canard lift. Implications on terminal velocity and time of flight using this mechanism to extend range are examined for various canard configurations. Performance predictions are conducted using a six-degree-of-freedom simulation model that has previously been validated against range data. The projectile dynamic equations are formed in the body frame and aerodynamic loads from the body and canards are Mach number and angle of attack dependent. The projectile body aerodynamic moments include unsteady aerodynamic damping. The focus of the study is directed toward low cost competent munitions that extend range and as such a simple flight control system is considered which utilizes only timer, roll rate, and roll attitude inputs.
\end{abstract}

Symbols: $x, y, z$ : Position vector components of the center of mass expressed in the inertial reference frame. $\phi, \theta, \psi$ : Euler roll, pitch, and yaw angles. $u, v, w$ : Translation velocity components of the center of mass resolved in the body reference frame. $p, q, r$ : Components of the angular velocity vector of the projectile expressed in the body reference frame. $X, Y, Z$ : Total external force components on the projectile expressed in the body reference frame. $L, M, N$ : Total external moments components on the projectile expressed in the body reference frame. $m$ : projectile mass. [I]: Mass moment of inertia matrix. $D$ : Projectile characteristic length. $C_{i}$ : Projectile aerodynamic coefficients. $q_{a}$ : Dynamic pressure at the projectile mass center. $\alpha$ : Longitudinal aerodynamic angle of attack. $\beta$ : Lateral aerodynamic angle of attack. $V$ : Magnitude of mass center velocity. $\phi_{C_{i}}$ : ith canard azimuthal angle. $\gamma_{C_{i}}$ : ith canard sweep angle. $\delta_{C_{i}}$ : ith canard pitch angle.

\footnotetext{
${ }^{1}$ Presented at the 9th Annual Gun Dynamics Symposium, November 17-19, 1998, McLean, Virginia.
}

\section{Introduction}

Traditionally, field artillery target effects are produced by firing a sufficient number of rounds with known accuracy at a given target so that, statistically, the target is neutralized. Generally, the goal of improving field artillery systems boils down to increasing achievable range and improving terminal accuracy of the projectile.

It is obvious that a round with extended range enjoys the flexibility of being able to engage a greater number of targets over a larger land area. A less obvious, yet equally important advantage of extended range is that the system with greater range requires fewer repositionings on the battlefield and can provide fire support for a higher percentage of time. This becomes particularly important in today's highly mobile battle environment, where a significant portion of time is spent moving into position. Since a long-range projectile can cover a large area of land, rounds can be fired at many different targets on the battlefield in a short period of time. Thus, a long range projectile adds increased flexibility to the field artillery commander, allowing him to influence a much greater portion of the battlefield, for longer periods of time.

The intensity of Army operations dictates that the field artillery optimize target effects with every round fired. One method of improving target effects is to increase the terminal accuracy of each round $[1,2]$. Current field artillery doctrine requires large numbers of rounds to be fired in order to neutralize a particular target. Statistically, only a small percentage of these rounds actually produce effects on the target. Increased accuracy provides that the 'extra' rounds, those not having effects on target, are never fired. If more rounds than necessary are fired, the firing unit accepts an unnecessary risk of being acquired by enemy target acquisition assets and subsequently engaged with counter fire. When the firing unit comes under steady counter fire, the unit will reposition and be rendered unavailable for other missions while in transit. Also, firing more than the minimum number of rounds needed to elimi- 
nate a target wastes expensive munitions and burdens the re-supply system. Every extra round expended on a particular target represents an opportunity cost that could have been directed at another objective on the battlefield. More importantly, target effects decrease exponentially as a function of the number of projectiles fired at a particular target due to the lack of surprise. The first volley fired is by far the most important volley to achieve maximum target effectiveness. Furthermore, if rounds are inaccurate, targets will reappear on the battlefield. Potentially, this will require additional artillery engagements and negatively affect the outcome of the overall battle plan. Thus, increased terminal accuracy increases target effects by landing more first hits, reduces vulnerability by firing less shots, and uses less material, which reduces the burden on the supply system - all contributing to a more effective and lethal field artillery unit.

The notion of utilizing canards as an aerodynamic control mechanism is by no means new and has been successfully applied to aircraft and missiles for some time. A significant aerodynamic database has amassed on aerodynamic modeling of missile configurations with canards [3]. However, few studies have specifically addressed the use of canards to extend the range of a ballistic projectile. Smith, Smith, and Topliffe [4] studied the use of canards on a spin stabilized projectile with the intended use of accuracy improvement. The relatively small canards were controlled by a seeker. To reduce the required control moments and actuator bandwidth, the canards were mounted on a bearing spinning at a different rate than the main body.

The work reported here evaluates the potential of extending the range of a field artillery projectile using moveable canards. It begins with a description of the simulation model used to predict projectile motion. This is followed with the definition of a representative extended range projectile configuration. With this model data, a parametric study is conducted which includes different canard geometry and deflection profiles.

\section{Projectile dynamic model}

The mathematical model describing projectile motion admits 6 rigid body degrees of freedom comprised of three body inertial position coordinates as well as three Euler angle body attitudes. The equations presented below use the ground surface as an inertial reference frame. The body frame is defined in the con- ventional manner [5] and the dynamic equations are written with respect to this coordinate system. The projectile translation and rotation kinematic and dynamic equations are given by Eqs (1) through (4) $[5,6]$.

$$
\begin{aligned}
& \left\{\begin{array}{c}
\dot{x} \\
\dot{y} \\
\dot{z}
\end{array}\right\}=\left[\begin{array}{cc}
c_{\theta} c_{\psi} & s_{\phi} s_{\theta} c_{\psi}-c_{\theta} s_{\psi} \\
c_{\theta} s_{\psi} & s_{\phi} s_{\theta} s_{\psi}+c_{\theta} c_{\psi} \\
-s_{\theta} & s_{\phi} c_{\theta}
\end{array}\right. \\
& \left.\begin{array}{c}
c_{\phi} s_{\theta} c_{\psi}+s_{\phi} s_{\psi} \\
c_{\phi} s_{\theta} s_{\psi}-s_{\phi} c_{\psi} \\
c_{\phi} c_{\theta}
\end{array}\right]\left\{\begin{array}{l}
u \\
v \\
w
\end{array}\right\} \\
& \left\{\begin{array}{l}
\dot{\phi} \\
\dot{\theta} \\
\dot{\psi}
\end{array}\right\}=\left[\begin{array}{ccc}
1 & s_{\phi} t_{\theta} & c_{\phi} t_{\theta} \\
0 & c_{\phi} & -s_{\phi} \\
0 & s_{\phi} / c_{\theta} & c_{\phi} / c_{\theta}
\end{array}\right]\left\{\begin{array}{l}
p \\
q \\
r
\end{array}\right\} \\
& \left\{\begin{array}{c}
\dot{u} \\
\dot{v} \\
\dot{w}
\end{array}\right\}=\left\{\begin{array}{c}
X / m \\
Y / m \\
Z / m
\end{array}\right\} \\
& -\left[\begin{array}{ccc}
0 & -r & q \\
r & 0 & -p \\
-q & p & 0
\end{array}\right]\left\{\begin{array}{l}
u \\
v \\
w
\end{array}\right\} \\
& \left\{\begin{array}{c}
\dot{p} \\
\dot{q} \\
\dot{r}
\end{array}\right\}=[I]^{-1}\left[\left\{\begin{array}{l}
L \\
M \\
N
\end{array}\right\}\right. \\
& \left.-\left[\begin{array}{ccc}
0 & -r & q \\
r & 0 & -p \\
-q & p & 0
\end{array}\right][I]\left\{\begin{array}{l}
p \\
q \\
r
\end{array}\right\}\right]
\end{aligned}
$$

where,

$$
[I]=\left[\begin{array}{ccc}
I_{R} & 0 & 0 \\
0 & I_{P} & 0 \\
0 & 0 & I_{P}
\end{array}\right]
$$

As shown in Eq. (6), the total applied force is composed of weight $(W)$, body aerodynamic force $(A)$, and canard aerodynamic force $(C)$.

$$
\left\{\begin{array}{l}
X \\
Y \\
Z
\end{array}\right\}=\left\{\begin{array}{l}
X_{W} \\
Y_{W} \\
Z_{W}
\end{array}\right\}+\left\{\begin{array}{c}
X_{A} \\
Y_{A} \\
Z_{A}
\end{array}\right\}+\left\{\begin{array}{c}
X_{C} \\
Y_{C} \\
Z_{C}
\end{array}\right\}
$$

The weight portion of the external loads is given by Eq. (7),

$$
\left\{\begin{array}{l}
X_{W} \\
Y_{W} \\
Z_{W}
\end{array}\right\}=m g\left\{\begin{array}{c}
-s_{\theta} \\
x_{\phi} c_{\theta} \\
c_{\phi} c_{\theta}
\end{array}\right\}
$$

while the aerodynamic force contribution is given by Eq. (8). 


$$
\begin{aligned}
\left\{\begin{array}{l}
X_{A} \\
Y_{A} \\
Z_{A}
\end{array}\right\}= & q_{a} D \\
& \left\{\begin{array}{l}
C_{X 0}+C_{X A 2} \alpha^{2}+C_{X B 2} \beta^{2} \\
C_{Y B 1} \beta+C_{Y B 3} \beta^{3}+C_{Y B 5} \beta^{5} \\
C_{Z A 1} \alpha+C_{Z A 3} \alpha^{3}+C_{Z A 5} \alpha^{5}
\end{array}\right\}
\end{aligned}
$$

The longitudinal and lateral aerodynamic angles of attack are computed using Eq. (9).

$$
\alpha=\tan ^{-1}\left(\frac{w}{u}\right) \quad \beta=\tan ^{-1}\left(\frac{v}{u}\right)
$$

The aerodynamic coefficients in Eq. (10) are functions of the local Mach number at the projectile mass center. They are computed using linear interpolation from a table of data.

The aerodynamic force due to a single canard is modeled as a point force acting at the lifting surface aerodynamic center. Canard orientation is defined by azimuthal angle $\left(\phi_{C_{i}}\right)$, sweep angle $\left(\gamma_{C_{i}}\right)$, and pitch angle $\left(\delta_{C_{i}}\right)$. In this study, canard pitch angle is controlled during flight. The $i t h$ canard force is described by Eq. (10),

$$
\begin{aligned}
\left\{\begin{array}{l}
X_{C_{i}} \\
Y_{C_{i}} \\
Z_{C_{i}}
\end{array}\right\}= & q_{C_{i}} S_{i}\left[T_{C_{i}}\right] \\
& \left\{\begin{array}{c}
C_{L} s_{\alpha_{i}-\delta_{i}}-C_{D} c_{\alpha_{i}-\delta_{i}} \\
0 \\
-C_{L} c_{\alpha_{i}-\delta_{i}}-C_{D} s_{\alpha_{i}-\delta_{i}}
\end{array}\right\}
\end{aligned}
$$

where $q_{C_{i}}$ is the dynamic pressure at the canard computation point, $S_{i}$ is the canard reference area, and $T_{C_{i}}$ is the transformation from the local canard reference frame to the projectile body frame. The canard lift and drag coefficients are expanded in terms of canard angle of attack and Mach number.

$$
\begin{aligned}
& C_{L}=C_{L 1} \alpha_{i}+C_{L 3} \alpha_{i}^{3}+C_{L 5} \alpha_{i}^{5} \\
& C_{D}=C_{D 0}+C_{D 2} \alpha_{i}^{2}+C_{I} C_{L}^{2}
\end{aligned}
$$

The coefficients in Eqs (11) and (12) are Mach number dependent. Canard angle of attack is computed in the same manner as the body angle of attack except the local relative velocity at the canard computation point is used.

The right hand side of the rotation kinetic equations contains the externally applied moments. The external moment components are given by Eq. (13) and contain contributions from steady body $(S A)$, unsteady body $\left(U_{A}\right)$, and canard $\left(_{C}\right)$ aerodynamics.

$$
\left\{\begin{array}{l}
L_{A} \\
M_{A} \\
N_{A}
\end{array}\right\}=\left\{\begin{array}{l}
L_{S A} \\
M_{S A} \\
N_{S A}
\end{array}\right\}+\left\{\begin{array}{l}
L_{U A} \\
M_{U A} \\
N_{U A}
\end{array}\right\}+\left\{\begin{array}{l}
L_{C} \\
M_{C} \\
N_{C}
\end{array}\right\}
$$

The steady body aerodynamic moment is computed by a cross product between the distance vector from the center of gravity to the center of pressure and the steady body aerodynamic force vector above. Like the aerodynamic coefficients, the center of pressure location is dependent on local Mach and is computed by linear interpolation. The unsteady body aerodynamic moment provides a damping source for projectile angular motion and is given by Eq. (14).

$$
\left\{\begin{array}{l}
L_{U A} \\
M_{U A} \\
N_{U A}
\end{array}\right\}=q_{a} D^{2}\left\{\begin{array}{c}
C_{D D}+\frac{p D C_{L P}}{2 V} \\
\frac{q D C_{M Q}}{2 V} \\
\frac{r D C_{M Q}}{2 V}
\end{array}\right\}
$$

The canard moment is computed through a cross product operation similar to the steady body aerodynamic moment. Air density is computed using the center of gravity position of the projectile in concert with the standard atmosphere [7].

The mathematical model described above has been validated against spark range data for a generic $25 \mathrm{~mm}$ fin stabilized sabot launched projectile [8]. Agreement between the model and range data is excellent.

\section{Configuration data}

Consider a representative extended range field artillery projectile that is fired from a $155 \mathrm{~mm}$ cannon. The projectile is $6 \mathrm{ft}$. long and weighs $120 \mathrm{lb}$. The projectile is equipped with pop-out rear fins and forward canards. The mass center is located $2.75 \mathrm{ft}$. from the base.

The body aerodynamic coefficients are shown in Figs 1 through 3 while the canard aerodynamic data is shown in Figs 4 and 5. Various size canards are considered below, however, all canard configurations share the same aerodynamic properties shown in Figs 4 and 5 .

\section{Simulation results}

Figures 6 through 13 compare the nominal trajectory and the extended range trajectory for the projectile described above. Range extension is accomplished with 4 equally spaced canards, each with a reference area of $0.07 \mathrm{ft}^{2}$ or a reference area ratio of 0.35 . A reference area ratio of 0.7 represents a practical upper limit on what could be installed in a production round. The initial forward body velocity is $750 \mathrm{~m} / \mathrm{s}$, the initial roll rate 


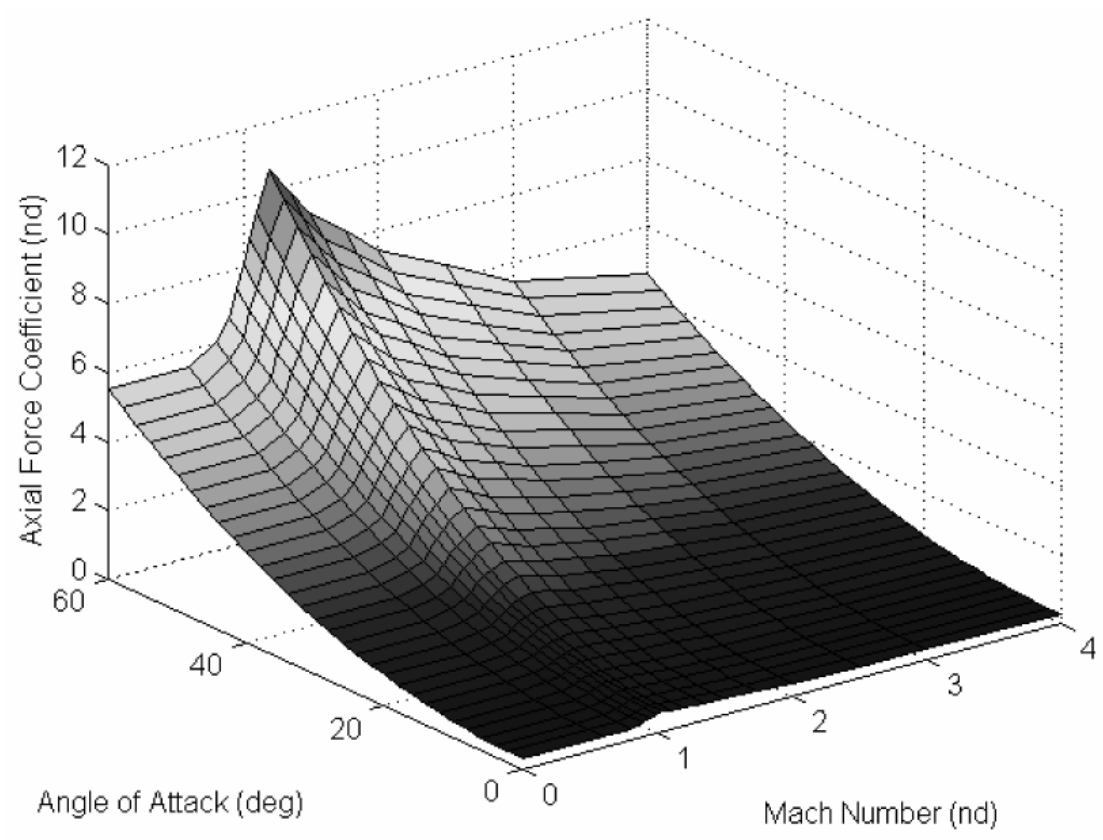

Fig. 1. Body axial force coefficient (nd).

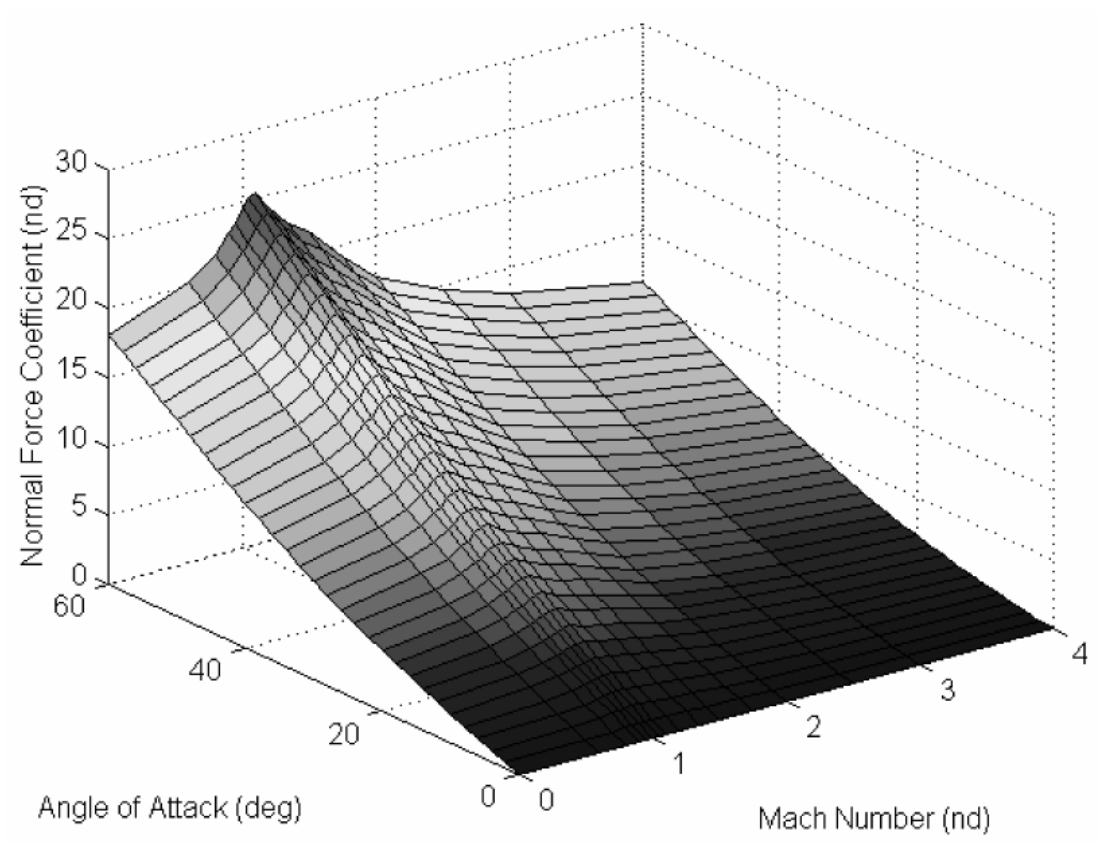

Fig. 2. Body normal force coefficient (nd)

is $31 \mathrm{rad} / \mathrm{s}$, and the gun elevation is 45 degrees. The fins are slightly canted to provide a slowly rolling projectile in steady state. For the range extension trajectory, a proportional plus integral (PI) roll control system is used prior to canard activation. Both canards in the body pitch plane are activated at the apex of the trajectory to an angle of $6 \mathrm{deg}$. Figure 6 compares range with and without movable canards installed. Under the conditions mentioned above, range is more than doubled from $17.5 \mathrm{~km}$ to $39.9 \mathrm{~km}$. The small discrepancy be- 


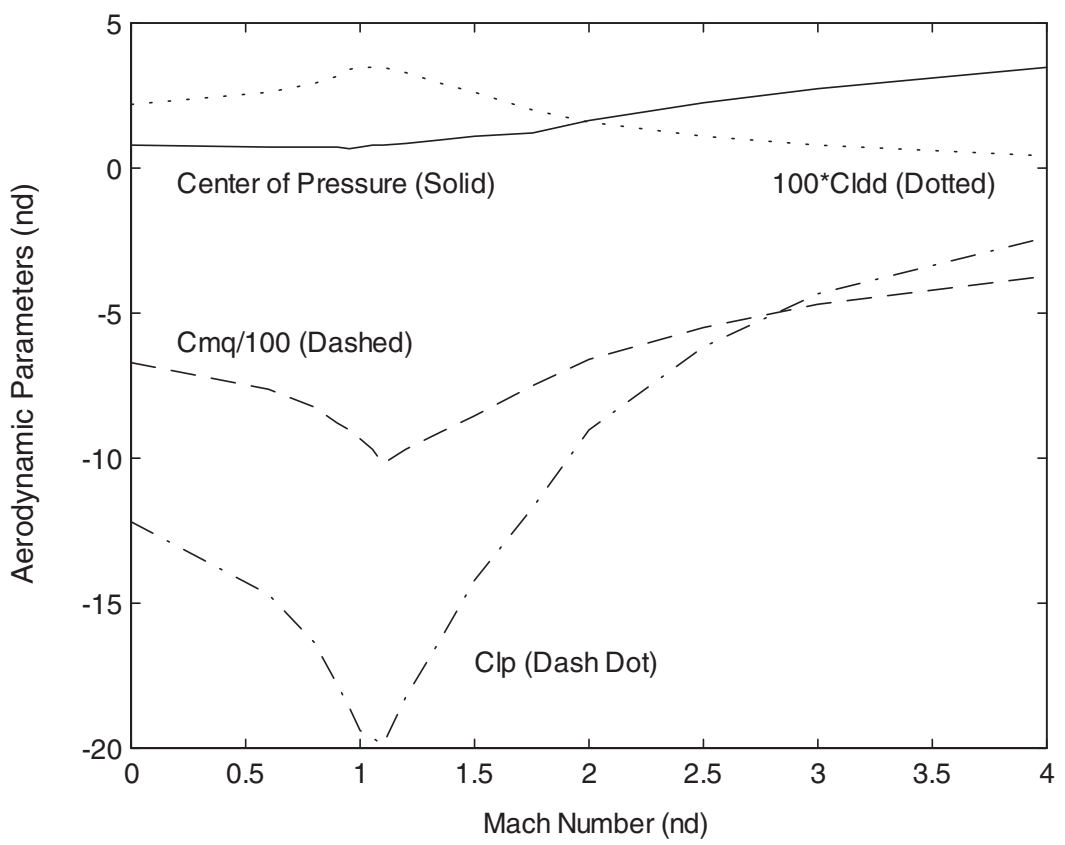

Fig. 3. Body aerodynamic parameters.

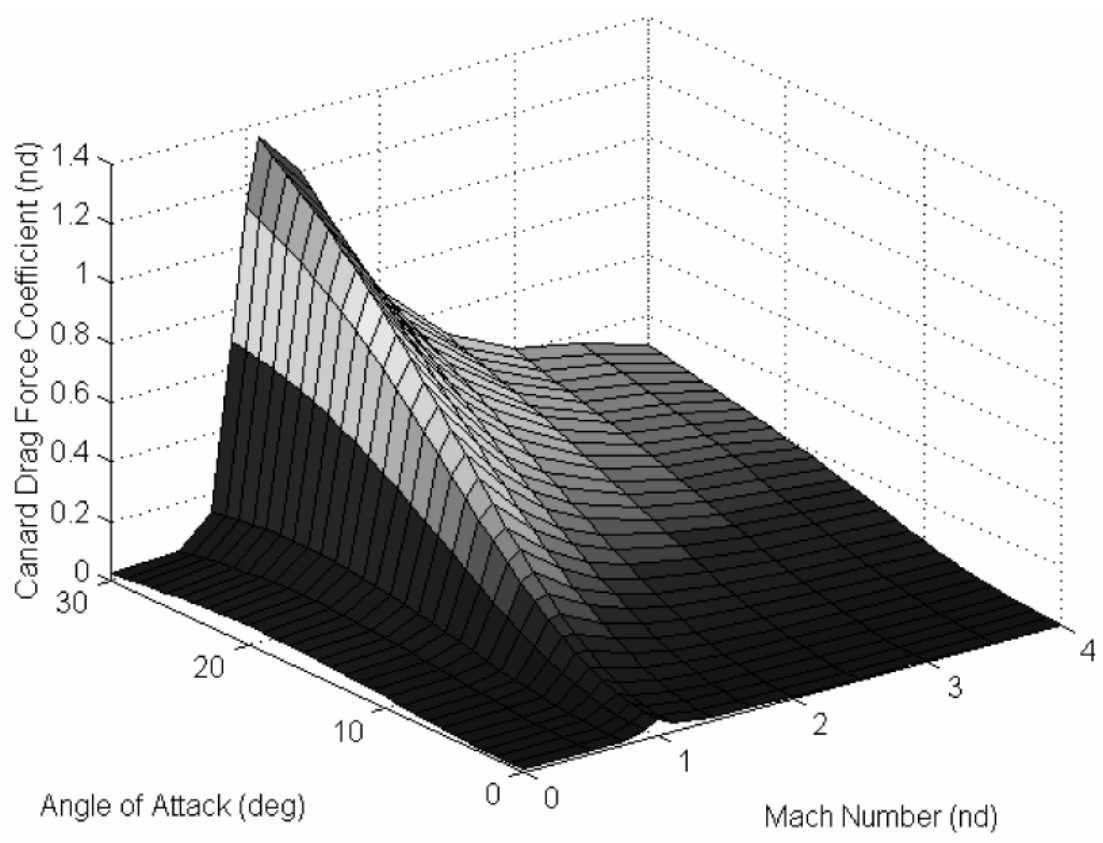

Fig. 4. Canard drag force coefficient (nd).

tween the nominal and canard configuration trajectories before the onset of the canard activation is due to drag of the canards, which are not present on the nominal projectile. From the altitude time history, it can be seen that the time of flight of the canard controlled projectile dramatically increases from 71 to $195 \mathrm{sec}$. Subsequent to canard pitch activation, a slight steady state yaw rate develops that alters the azimuth of the projectile hence creating a difference in the cross range between the two trajectories. As shown in Fig. 8, both the baseline 


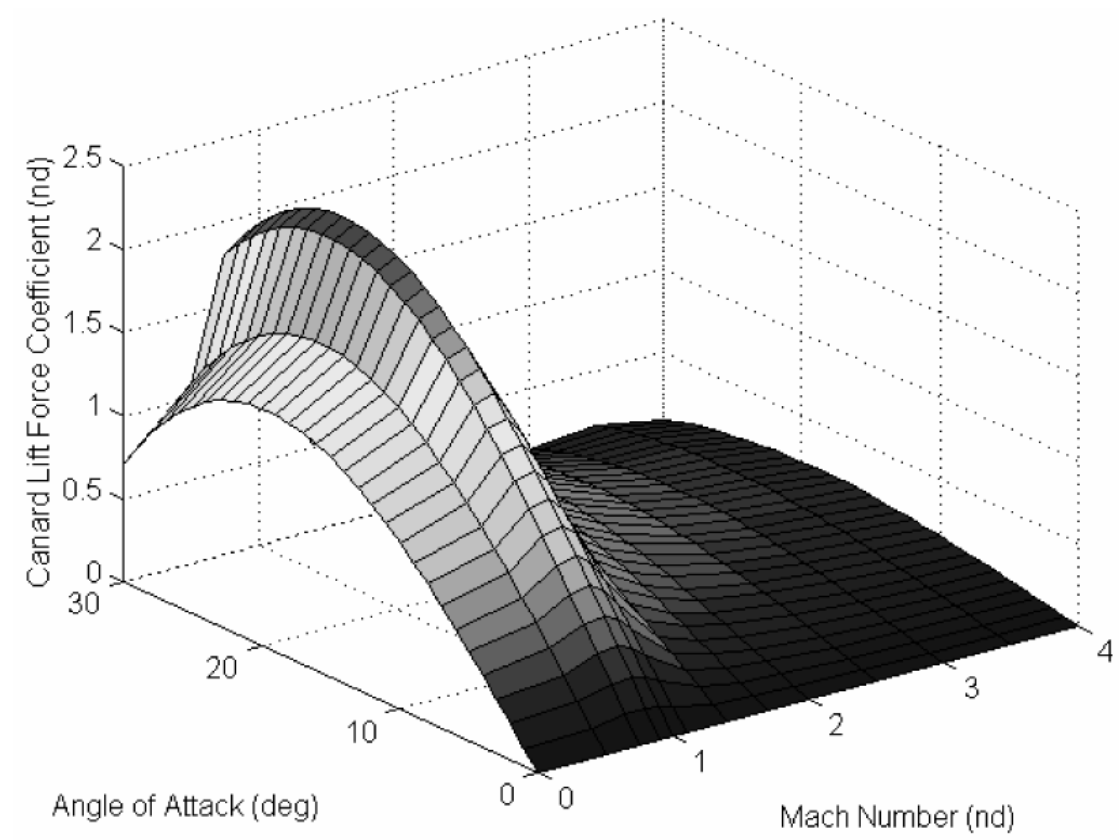

Fig. 5. Canard lift force coefficient (nd).

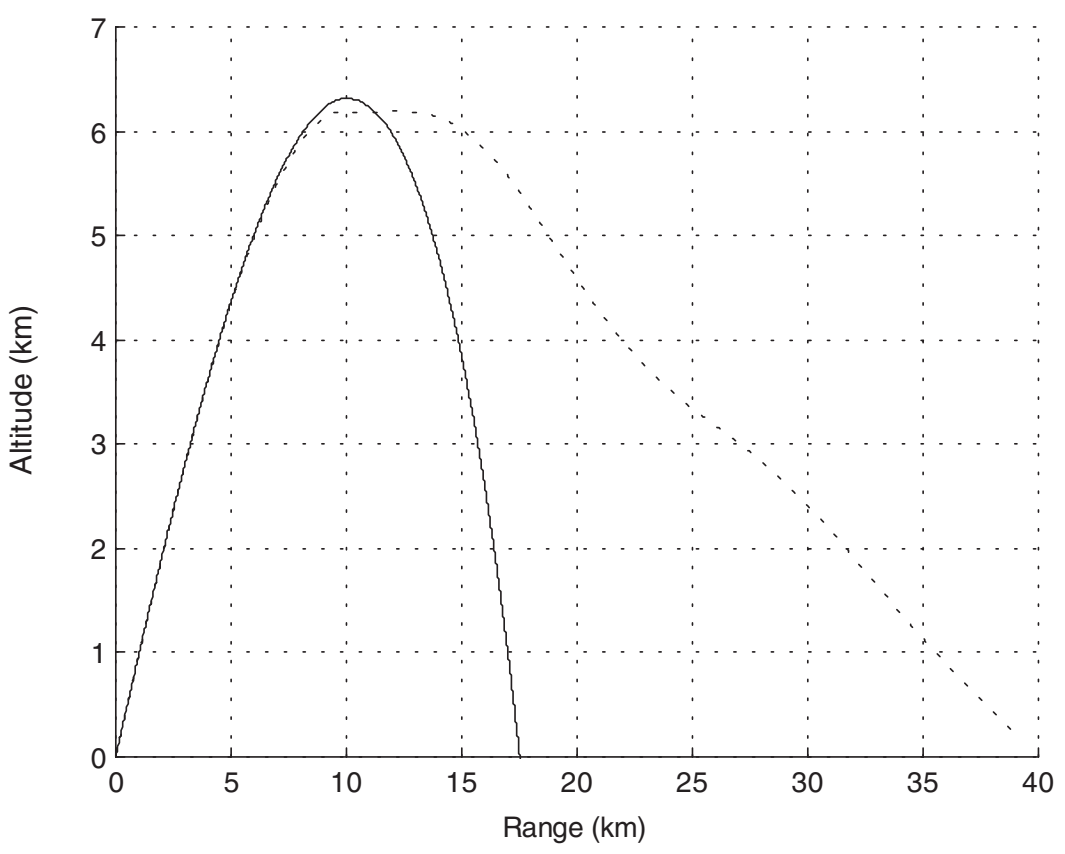

Fig. 6. Altitude vs. range $($ Solid $=$ Nominal, Dotted $=$ Extended $)$.

and controlled trajectories have a $45 \mathrm{deg}$ pitch attitude at launch; however, the baseline trajectory pitch attitude monotonically decreases with time, whereas the controlled trajectory experiences some transient oscillations immediately after the pitch command is exe- cuted. After transient oscillations settle, pitch attitude of the controlled trajectory approaches a value of -10 deg. The forward velocity time history, given in Fig. 9, shows the nominal trajectory impacts the target with a velocity of $300 \mathrm{~m} / \mathrm{sec}$ compared to the controlled tra- 


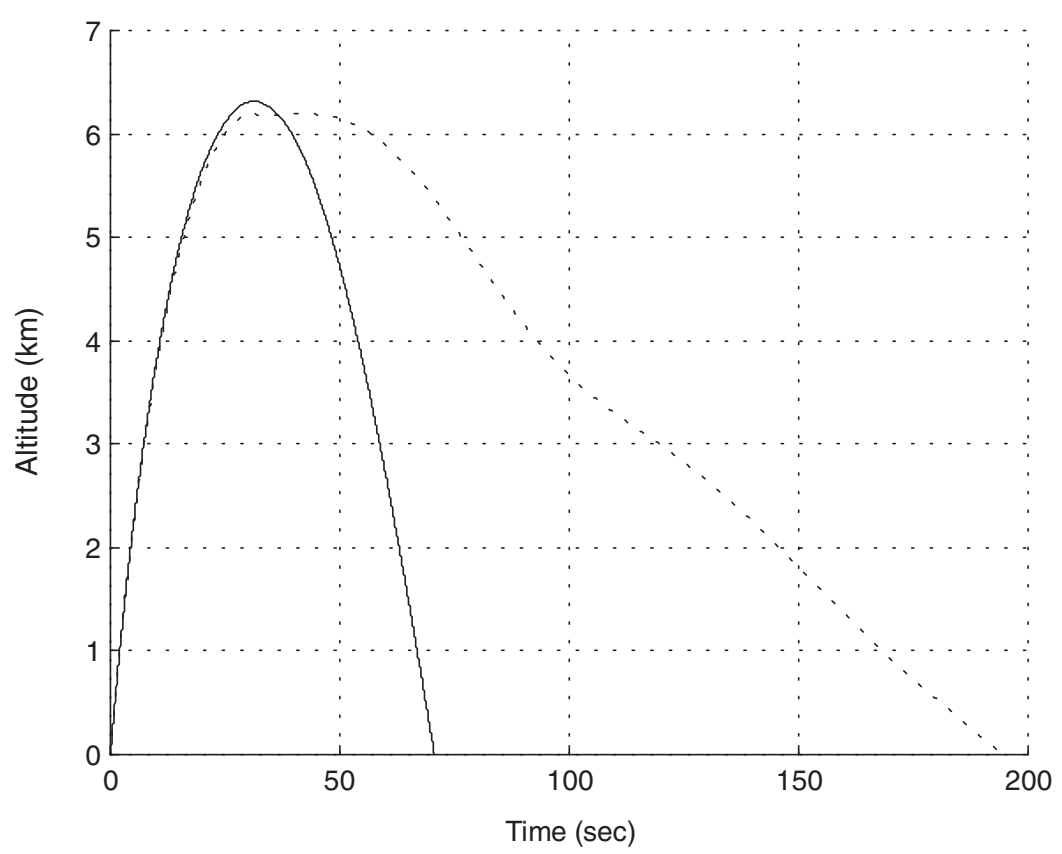

Fig. 7. Altitude vs. time (Solid $=$ Nominal, Dotted $=$ Extended $)$.

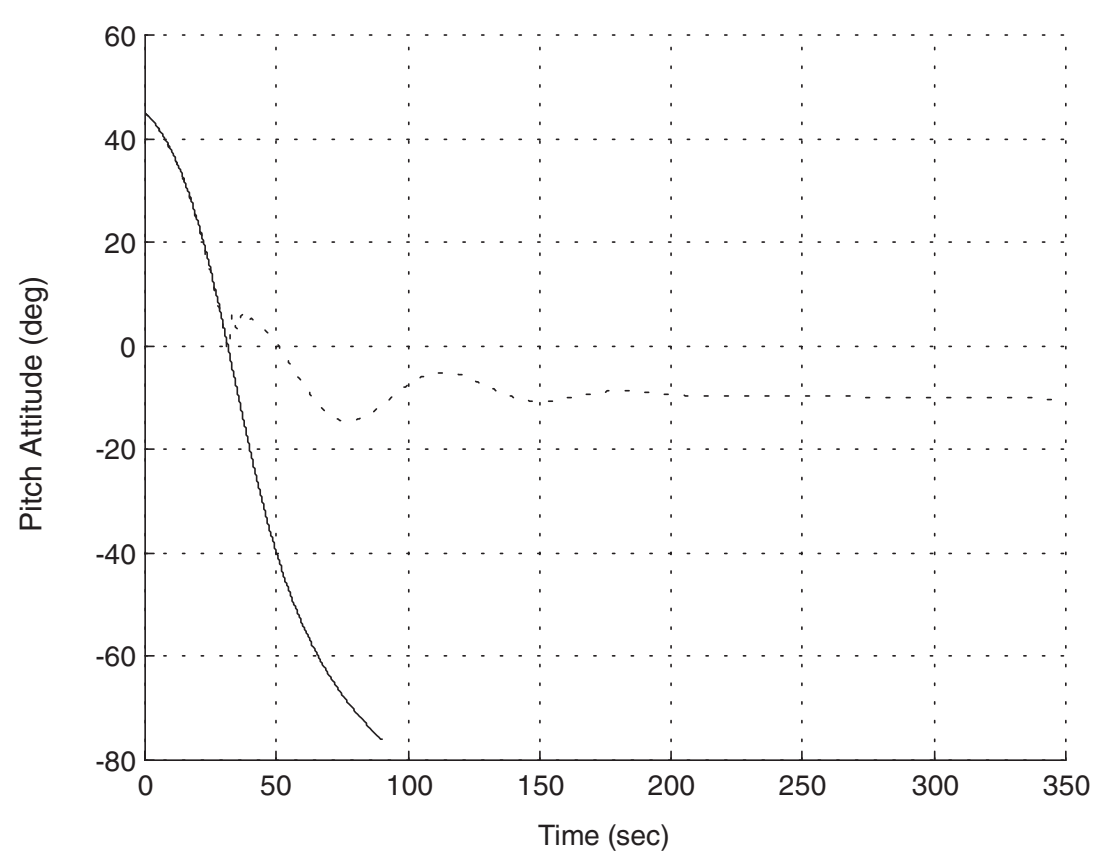

Fig. 8. Pitch attitude vs. time (Solid $=$ Nominal, Dotted $=$ Extended $)$.

jectory at a velocity of $166 \mathrm{~m} / \mathrm{sec}$. Furthermore, the reduced velocity shown in the controlled trajectory is present throughout a large portion of the flight. The roll rate time history, given in Fig. 10, shows the PI roll control system eliminates roll rate from $31 \mathrm{rad} / \mathrm{sec}$ to 0 within $5 \mathrm{sec}$ whereas the baseline trajectory has a steady state roll rate due to rear fin cant. Roll angle control is vital for this range extension strategy, since the pitch attitude commands assume the projectile will maintain near zero roll angle from the start of pitch 


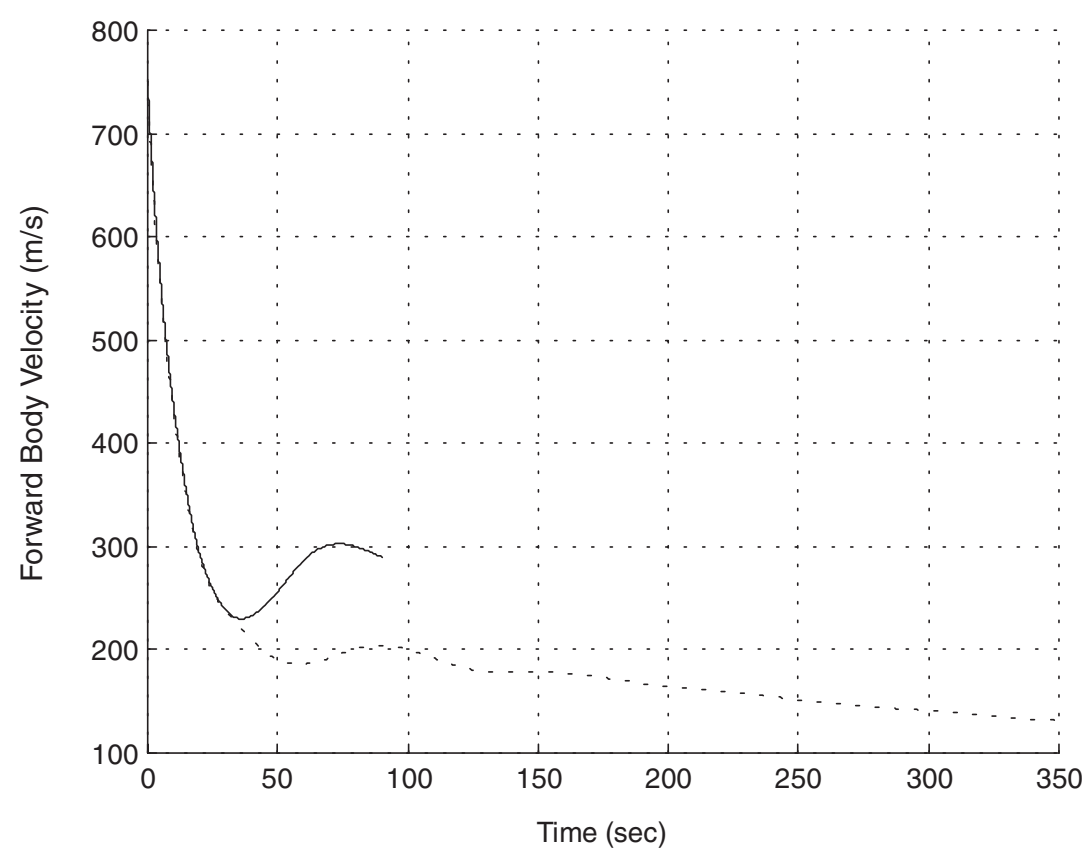

Fig. 9. Forward body velocity vs. time $($ Solid $=$ Nominal, Dotted $=$ Extended $)$.

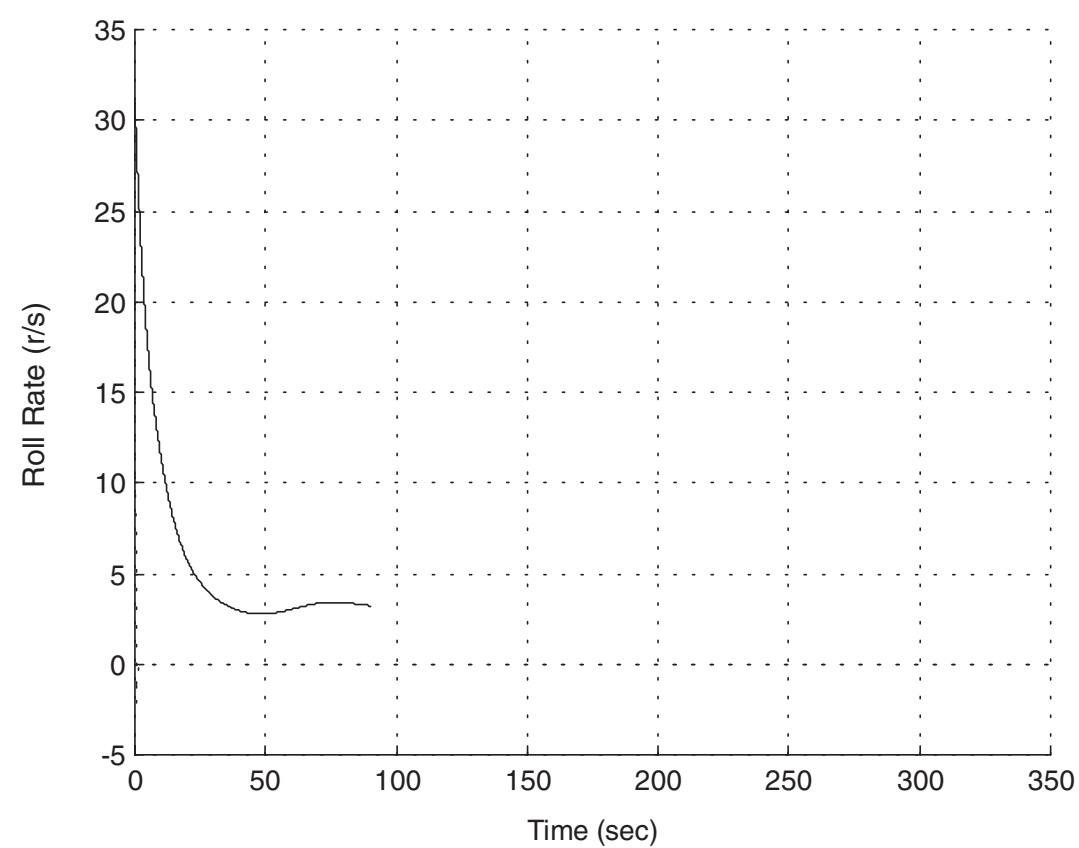

Fig. 10. Roll rate vs. time $($ Solid $=$ Nominal, Dotted $=$ Extended $)$

commands to the terminal point. Figure 11 shows the total aerodynamic angle of attack of the projectile for both the nominal and controlled case. The nominal angle of attack is small, less than half a deg. However, the canards induce maximum angle of attack oscillations slightly over $7 \mathrm{deg}$ and a steady state glide angle of attack of $4.1 \mathrm{deg}$. As canard angle of attack essentially mimics body angle of attack, the canards can stall during the transient part of the response. Figures 6 through 11 show a dramatic extension of range using body and 


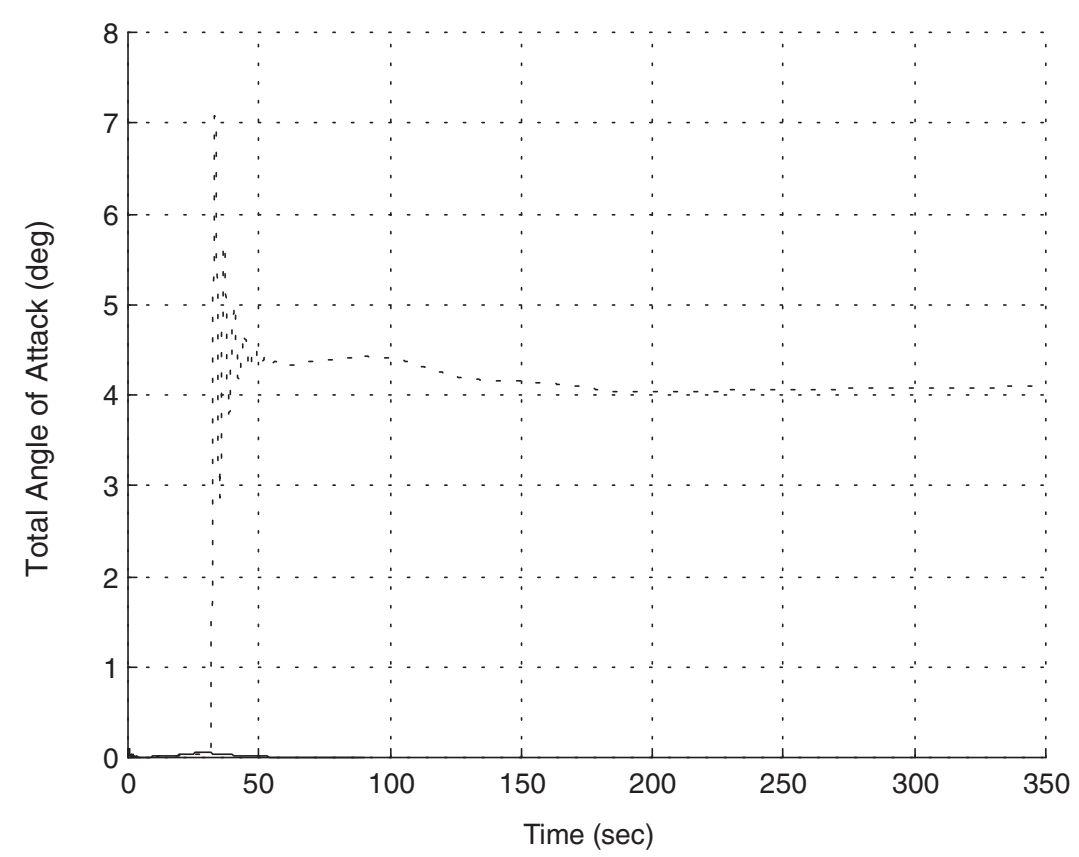

Fig. 11. Total angle of attack vs. time $($ Solid $=$ Nominal, Dotted $=$ Extended $)$.

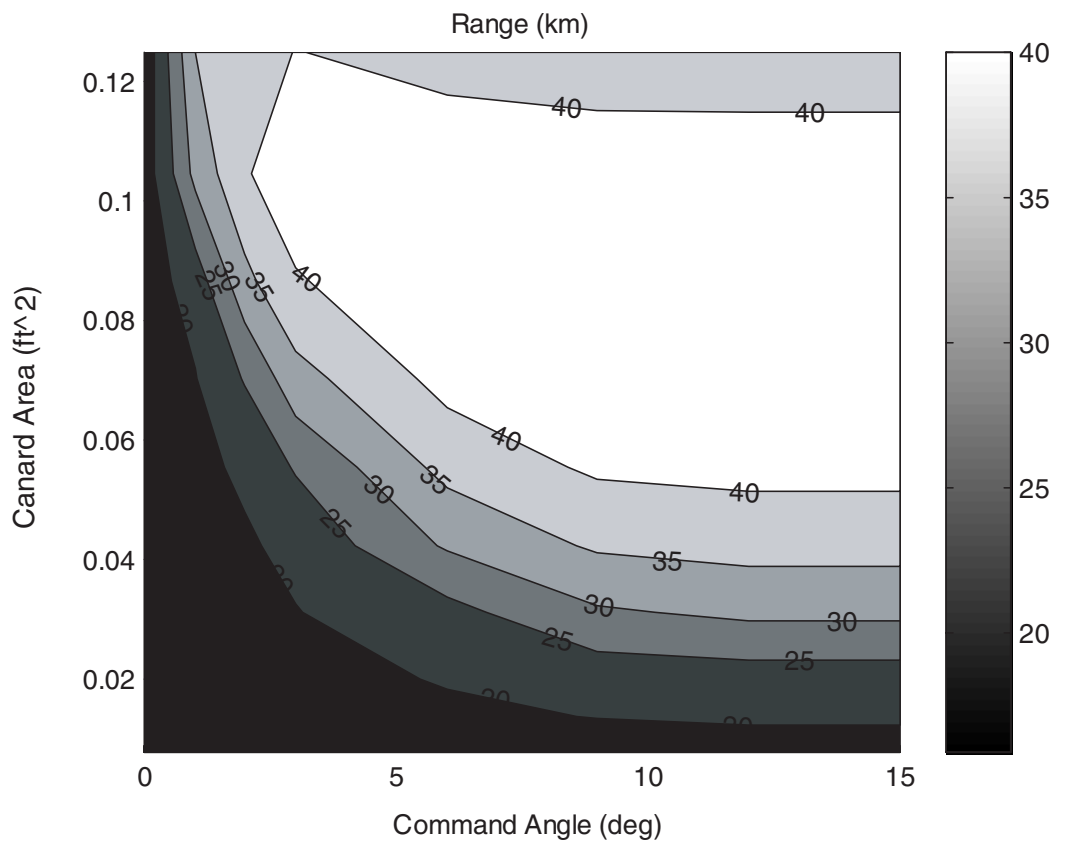

Fig. 12. Range vs. canard area and canard command angle.

canard lift that is achieved by pitching the projectile upward using movable canard lifting surfaces. While a dramatic increase in range is achievable, an equally dramatic increase in the time of flight and decrease in the impact point velocity is realized. Thus, the action of the moveable canards is to facilitate a transfer of kinetic energy to potential energy, allowing the projectile to stay aloft longer.

Figures 12 through 14 show range, flight time, and terminal velocity for various canard areas and various 


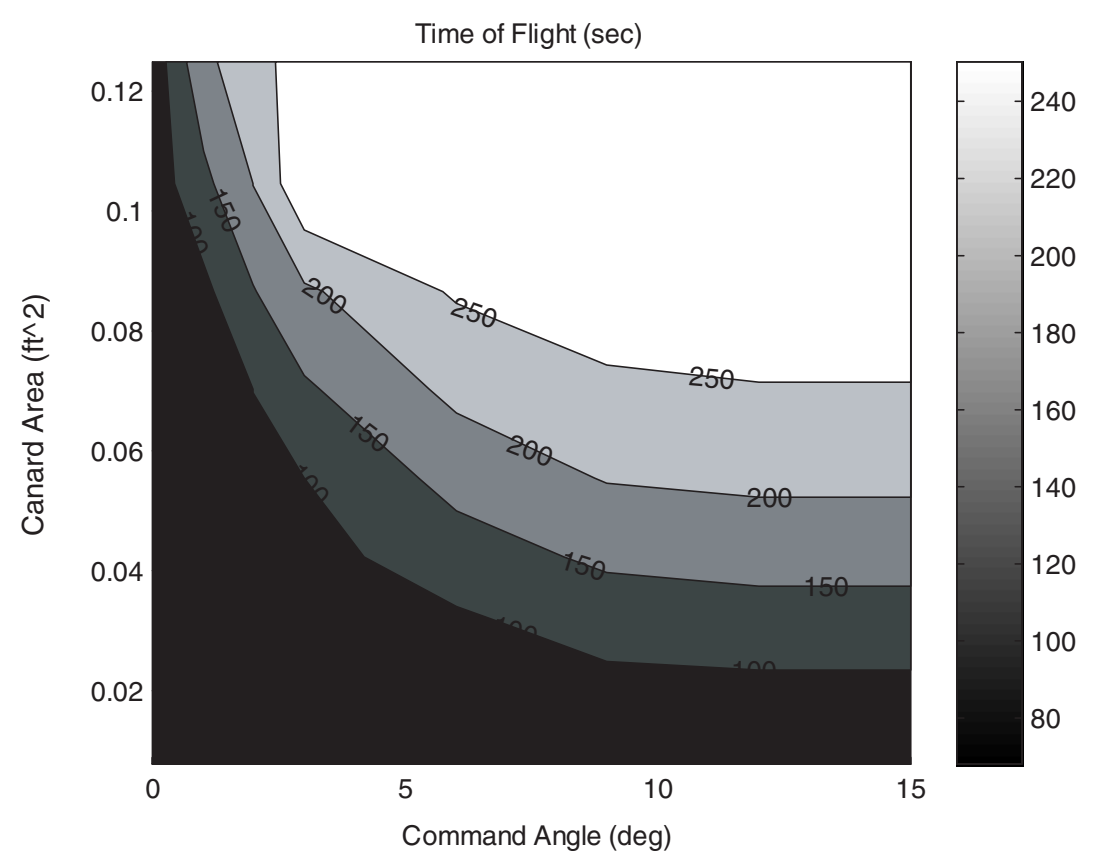

Fig. 13. Flight time vs. canard area and canard command angle.

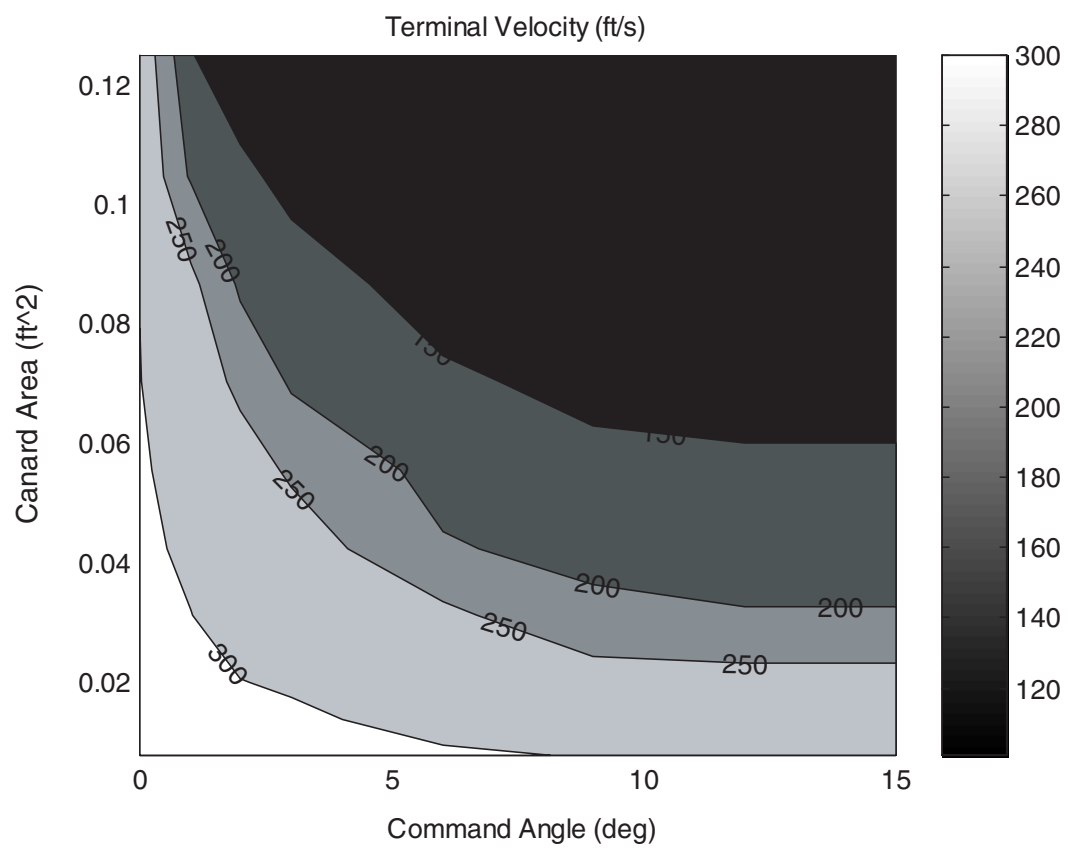

Fig. 14. Terminal velocity vs. canard area and canard command angle.

canard command angles. For all data points in Figs 12 through 14, the canard command was executed at the apex of flight. As would be intuitively expected, increasing canard area or the canard command angle increases range. However, when canard area is relatively large, increasing canard command angle tends to reduce range due to angle of attack vibration, which leads to higher projectile drag. Also, for a given canard area, flight time is approximately constant for large command angles. 


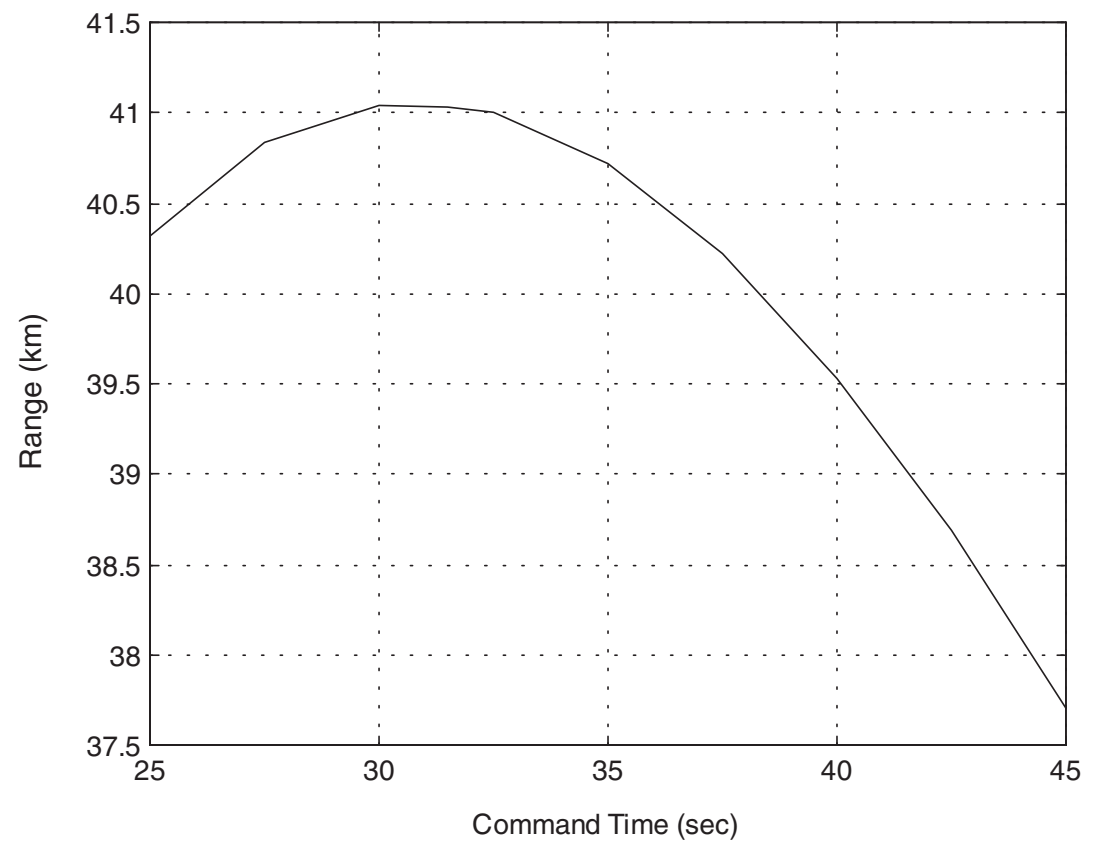

Fig. 15. Range vs. canard command activation time.

Figure 15 shows range versus time of canard activation. For the case shown, the apex of flight was reached 31.5 seconds into flight. Figure 15 shows that for maximum range, the canard command should be executed at the apex of flight. Also, the curve is relatively symmetric about the maximum range point.

\section{Conclusions}

Using a six-degree-of-freedom projectile trajectory simulation model driven by projectile data representative of an indirect fire field artillery round, it is found that a small set of four canards can dramatically extend projectile range. The side effects of increased range using this mechanism are: decreased terminal velocity, increased flight time, increased nose-up terminal pitch attitude, and increased aerodynamic angle of attack. Moreover, maximum range is attained when the canards are activated at the apex of the trajectory.

\section{References}

[1] R.W. Pointer and C.E. Dean, Cannon Artillery May One Day Soon Provide Crisis Logistics To Ground Forces, National DEFENSE LXXX(518) (May 1996).

[2] C.E. Dean and W.P. Wiesman, Combat Resupply by Artillery, Army RD\&A PB70-96-1 (January 1996).

[3] USAF Stability and Control DATCOM, AFFDL Wright Patterson AFB, Ohio, November 1965.

[4] J.A. Smith, K.A. Smith and R. Topliffe, Feasibility Study for Application of Modular Guidance and Control Units to Existing ICM Projectiles, U.S. Army Armament Research and Development Command, Contractor Report ARLCD-CR-79001, November 1978.

[5] B. Etkin, Dynamics of Atmospheric Flight, John Wiley and Sons, New York, 1972.

[6] C. Murphy, Free Flight of Symmetric Missiles, Ballistics Research Laboratory Report BRL-1216, Aberdeen Proving Ground, Maryland, 1963.

[7] R. Von Mises, Theory of Flight, Dover Publications Inc., New York, 1959.

[8] M. Costello and D. Anderson, Effect of Internal Mass Unbalance on the Stability and Terminal Accuracy of a Field Artillery Projectile, Proceedings of the 1996 AIAA Atmospheric Flight Mechanics Conference, San Diego, CA, 1996. 

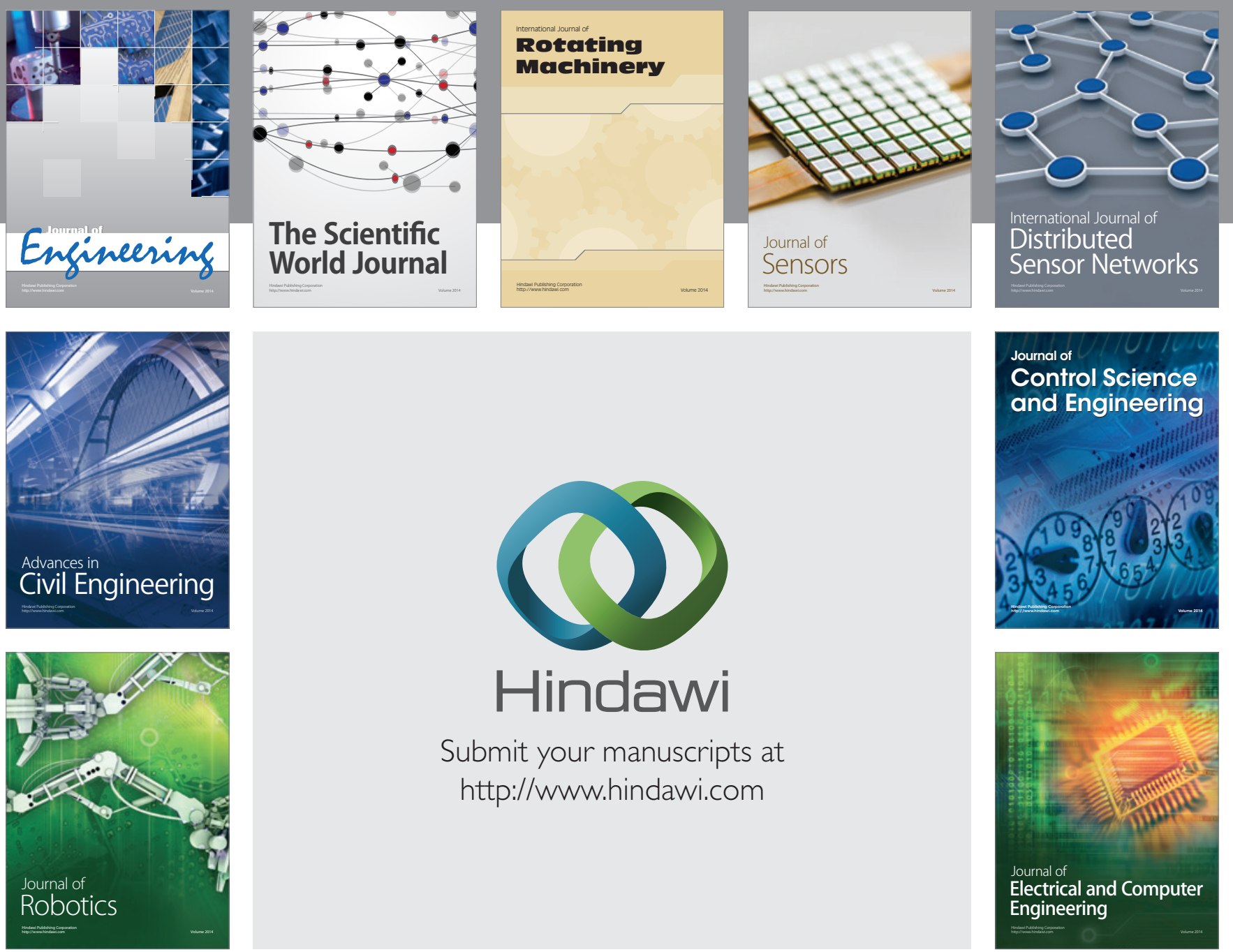

Submit your manuscripts at

http://www.hindawi.com
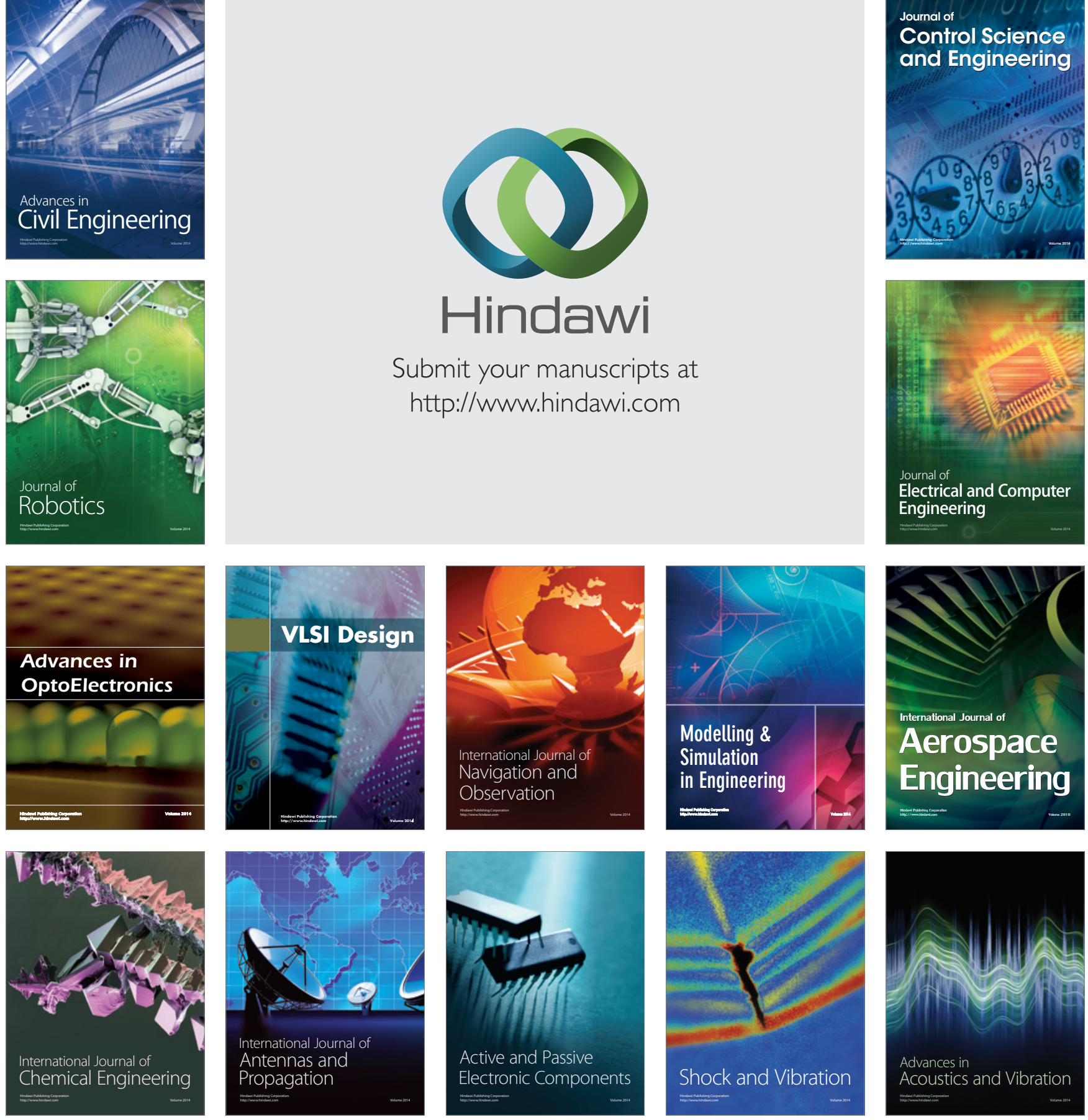